\title{
Keyword Index for Volume 97
}

$1 \mathrm{p} 1416$

3-phosphoinositide-dependent protein kinase-1 1465

3D tumour model 194

5-fluorouracil 334, 910, 919, 1028,1532

adaptive conjoint analysis 717 adenomatous polyposis coli 384 adenosarcoma 1194

adenovirus 992

adjuvant chemotherapy 654 , 1021

adjuvant; neutropaenia 1642

adolescent 29

adrenal tumour 420

adult T-cell leukaemia 1099

advanced cancer 177, 1475

advanced ovarian cancer 1200

aerobic glycolysis 646

aetiology 1287, 1291

Affymetrix 1165

ageing 1189

AKT 218, 785, 809

albumin 1266

alcohol 118, 429, 700, 1287

alkaline sphingomyelinase 1441

ALL 992

allelic imbalance 1157

AML 877

anastrozole 152

androgen-independent 1206

androgens 688

androstene neuro-steroids 619

aneuploidy 1218

angiogenesis $391,513,1277$,

$1432,1523,1673$

angiopoietin family 877

anilinoquinazolines 183

anti-adhesion 730

anti-EGFR treatment options 92

anti-folates 1071

antiangiogenic 65

anticancer agent 1344

antigen shedding 1146

antihypertensive treatment 112

antimetabolites 628

anxiety 1625

APC antibody specificity 384

apoptosis 253, 277, 637, 769,

$902,1077,1225,1234,1432$, 1532, 1696

aromatase 755

aromatase inhibitor 152

array-CGH 260

asbestos 1300

aspirin 1295

attributable proportion 129

audiovisual patient information 705
Aurora-A 1664

autologous transplantation 539

awareness 691

AZD2171 65

B-CLL 769

basement membrane 1505

BCG-refractory 1499

Bfl-1 769

biliary tract cancer 1577

biochemical recurrence 678

biomarker 1, 1457

biopsy 952

bladder cancer 1499

blood outgrowth endothelial cell 513

blood pressure 112

blood testing 1399

blood-brain barrier 322

body mass 1486

body mass index 995, 1005

bone metastasis 964

bone tumours 1588

bone turnover 964

bortezomib 1099

brain tumour 1583

BRCA2 gene 826

breast and bladder cancer 502

breast cancer 58, 105, 152, 327, $434,440,479,654,686,688$, 725, 761, 809, 832, 957, 964, 978, 1046, 1090, 1157, 1175, $1211,1251,1277,1287,1361$, $1372,1505,1523,1570,1625$, $1632,1642,1673$

breast cancer susceptibility 1696 breast conservation 725,883 , 1046

breast surgery 1211

$\left[{ }^{11} \mathrm{C}\right]$ carvedilol 322

c-Kit 857

c-MET 368

C-reactive protein 1266

C4.4A 1146

C6 cells 755

$\mathrm{Ca}^{2+}$-binding protein 792

cancer clinical trials 705

cancer mortality 1300

cancer services 1588

cancer survivors 612

cancer survivorship 1625

cancer testis antigen 543

cancer vaccine 1381

capecitabine $712,862,1333$, 1475

carbonic anhydrases 1465

carboplatin 162

carcinogenesis 646

carcinoma of an unknown primitive 857

cardiotoxicity 1084 case-control study 429, 434, 995

caspases 1234

CCR7 670

CD9 941

CDK4 1242

CEA 745

celecoxib 1206

cell cycle 277, 1513

cell cycle checkpoints 1664

cell death 1381

cell migration 1361, 1432

cell-free DNA 1399

cell-of-origin subtypes 1165

central nervous system tumours 1588

cervical cancer $129,133,218$, 1322, 1457

cervical carcinoma 1058

cervix carcinoma 1135

CESH 1707

cetuximab 494, 1139

CGH 238

chemokine receptor 670

chemoprevention 327

chemoradiotherapy 712,883

chemoresistance 769

chemotherapy $29,862,1028$, $1040,1157,1234,1642$

Chernobyl 818

childhood acute lymphoblastic leukaemia 140

childhood cancer 412, 695, 986, 1583

childhood leukaemia 986, 1009, 1315

Chile 85

China 123, 1577

Chlamydia 949

cholecystitis 1577

chromosomal instability 1218

chromosome 1696, 1707

chronic lymphocytic leukaemia 253

cigarette smoking 700

circumferential resection margin 1333

cisplatin $162,194,712,851$, 1234,1329

clear cell adenocarcinoma 1053

clear cell renal cell carcinoma 420

clinical decision support systems 486

clinical phase II study 1084

clonality 1260

coffee 426, 1291

cognitive behaviour therapy 612

cohort $686,1295,1570$

cohort studies 115, 118, 123,

$129,426,1005,1291,1300$

collagen I 358

colon cancer 1021, 1655 colorectal adenoma 1425, 1449

colorectal cancer 297, 446, 745, 971, 978, 1028, 1035, 1139, $1146,1266,1305,1425,1486$, 1606

colorectal cancer cells 384

colorectal neoplasms 118, 1393, 1601

colorectal tumours 92

combination index 628

combination treatments 888

communication skills 472

comparative genomic

hybridisation 1707

computer simulation 1322

conformal radiation 464

convection 194

copper transporter ATP7A 334

cost-effectiveness analysis 152 , 1322

cost-utility analysis 152

Costa Rica 837

costs 479

COX-2 inhibitor 1206

COX2 557

coxibs 1465

CRH 637

CS-682 628

CXCL12 761

CXCR4 761

cyclooxygenase-2 1388, 1523

cyclooxygenase-2 independence 1465

cyclophosphamide 1200

CYP3A4 metabolism 440

cytokines 105, 210

cytotoxic T lymphocyte 1648, 1655

DDS 170

deep-vein thrombosis 1053

degradome 201

degranulating $\mathrm{CD} 8{ }^{+}$cells 1381

dendritic cells 1251

depression 1625

deprivation 999

detection methods 1632

diabetes 995

Dickkopf-1 964

dietary iron 118

diffusion 194

disease progression 1469

disease-free survival 50

Dkk-1 1552

DNA crosslinking 253, 927

DNA damage 1189

DNA hypomethylation 412

DNA methylation 895, 1457

DNA repair 334, 927

docetaxel 290, 593, 851, 1206,

$1338,1475,1613$

doctor discipline 6 
1726

doctor specialty 6

dose intensity 1642

dose-limiting toxicity 177

downstaging 851

doxorubicin 43, 1084

doxorubicin resistance 1077

drug delivery 194, 735, 910

drug development 577

drug resistance 562, 927, 1218

ductal carcinoma in situ (DCIS) 725

dynamic models 1322

dysplasia 1545

\section{E-cadherin 358}

E2F 1242

early-onset disease 826

EBV 1567

EGFR 453, 494, 659, 778, 857, 1139,1560

EGFR downstream signalling pathway 92

EGFR mutations 741, 1560

elderly 162,1606

ELISA 398

embryonic tumours 412

endometrial cancer 995, 1538, 1194

endoplasmic reticulum (ER) stress 1465

endoscopy 1493

endostatin 513

endovesical instillation 1499

England 989

ENPP7 1441

EpCAM (CD326) 315

epidemiology 112, 1287, 1583

epidermoid cancer 1234

epigenetic process 1116

epigenetics 1, 267, 562, 986, 1416

epirubicin 1200

ErbB2 659, 1354

ErbB2 mutation 741

ErbB3 453

ESCC 1409

esophageal cancer 218

estramustine 1206, 1613

etoposide 162

expectations 14

extracellular matrix 201

$\alpha$-fetoprotein 327

FACS 73

faecal occult blood 1601

Fas ligand 73, 637

fatigue 612

FDG-PET 902, 1493

fetal origins of disease 686

fibroblasts 210, 398

first-line treatment 297

fluorescent in situ hybridisation 1139

foetal haemoglobin 412

folate 1449

folate metabolism 247

FOLFIRI 1035

follow-up 1632
$\mathrm{G}_{1}$ phase 1242

galectin-3 1146

gallstones 1577

GAMEC 308

gastric adenocarcinoma 458

gastric cancer $37,123,543,550$,

$589,700,712,837,851,902$,

1493,1567

gastric cancer screening 837

gastric carcinoma 334

GDEPT 745

gefitinib 65, 183, 778, 1560

gemcitabine $43,145,283,464$, 1329, 1499

gene expression 145, 895

gene expression profiling 1432

gene fusion 1690

gene therapy 513

genetic alterations 260

genetic susceptibility 1701

genetics 1305

genomics 1116

germ cell 308

glioblastoma 302, 619

glioma cells 755

glucose transport 902

glucose-regulated protein $94 \mathrm{kDa} 792$

GLUT-1 646

glycosaminoglycan 761

glycosylation 1146

grapefruit intake 440

GSTP1 1399

Guthrie cards 992

haematological malignancies 1588

haeme oxygenase-1 1683

haplotype 832

HCC 1399

HCV 1399

HDAC 1344

HDAC inhibitor 177, 1344

head and neck cancer 65,670

hemangiosarcoma 115

heme iron 118

heme oxygenase-1 1099

hepatic resection 1606

hepatitis $C$ virus 426

hepatocellular carcinoma

(HCC) 50, 426, 582, 862, 1260,1532

HepG2 1441

HER dimerisation inhibitors 1338

HER-2 105, 453, 494

Her-2/neu overexpression 857

HER-3 453

hereditary 486

hetastatic breast cancer 1040

hexokinase 902

high-dose chemotherapy 308 , 391,1200

high-risk breast cancer 391

histone modifications 1

histopathology 1393

HLA-A3 supertype 1648

HMEC 1361
Hodgkin lymphoma 29

Hodgkin's 1310

homocysteine 1071

hormonal 1194

hormone replacement therapy

118,1486

hormone-refractory 378,1480

hormones 688

HPV 218

HPV infection 133

HPV vaccination 1322

HRQOL scores 302

Hsp90 741

human gastric cancer 895

human papillomavirus 85,691

hypercalcaemia 183

hypericin 502

hypermethylation 267

hypertension 995

hypomethylation 267

hypoxia 646

IBC signature 1165

Id proteins 1409

IL-6 378

IL-6R 378

imatinib 735

immune function 1251

immune privilege 637

immunohistochemistry 398 ,

405, 523, 531, 1135, 1404

immunostaining 952

immunotherapy $210,315,598$, 1354

immunotherapy CD40 1251

impact evaluation 837

improvement 1469

incidence 1009, 1567

individual dosing 290

indomethacin 1077

infection 1310

inflammatory breast cancer 659 , 883

inhibitor of apoptosis 1271

insulin 98

insulin-like growth factor 98

insulin-like growth factor

binding protein 98

integration 85

integrins 1090

interferon resistance 231

interferon- $\gamma 420$

interleukin-6 1513

international differences 1277

interphase FISH 678

interstitial fluid pressure 735

intervention 612

invasion 73, 358, 368, 1505

invasiveness 50

ionising radiation 1664

irinotecan 297, 593, 1035

irradiaton 322

isolated tumour cells 589

JAK 378

JNJ-16241199 1344
K-Ras 1139

K-ras/BRAF mutations 1425

Ki-67 1124

KIF2C 543

kinase inhibition 741

knowledge 691, 705

Lactobacillus rhamnosus GG 1028

late complication 1058

Lck 1648

left-handedness 686

letrozole 802

leucovorin 297

leukocytes 978

lifestyle factors 133

ligand interactions 1361

liver cancer 1005

liver diseases 1441

liver metastases 1035

liver tumour 1441

Livin/ML-IAP/KIAP 1271

local recurrence 1046

locally advanced rectal cancer 1333

locoregional relapse 1632

long-term effect 612

loss of heterozygosity 1157

lung cancer 85, 183, 247, 735, 1295,1300

lymphadenectomy 1194

lymphoma 539, 619, 1310

malignant glioma 619

malignant melanoma 231

mammary carcinoma 1354

marker 1545

Masaoka's staging system 22

mass screening 1601

mast cells 952

maternal 688

mathematical models 646, 1322

matrikine 1505

matrix metalloprotease 1106

matrix metalloproteinase 398,

971, 1505

maximum tolerated dose 177

MCAK 543

MCM-2 1124

MCM-5 1124

MDA-MB-231 1361

medical education 472

medulloblastoma 267

melanoma 223, 1225, 1329

melatonin 755

mesenchymal stem cell 1552

mesothelioma 1300

meta-analysis 1005, 1291

metastasis $223,670,761,868$,

957, 1409, 1523, 1606

metastatic breast cancer 1040

metastatic gastric cancer 593

methotrexate 308

methylation 1425

microarray 818

microdose 577

micrometastasis 550 
micronuclei 1218

microtubule 1218, 1673

microtubule inhibitors 888

microvessel density 391, 405, 1277

midkine 405

migration 543, 1090

minimum dataset 1393

mitochondria 345

mitogen-activated protein

kinase 659

mitomycin 1475

mitotic catastrophe 941

mitoxantrone 1613

MMP 957

modulation 919

molar pregnancy 986

monoHER 1084

monohydroxyethylrutoside 1084

monounsaturated fat 1570

morphine 1523

mortalin 941

mortality 1009

mortality trends 1588

motility 358

MRP1 1077

MS-MLPA 1457

Ms-SNuPE 1457

MSC 1552

MSP 1457

MT1-MMP 358

MUC-1 730

MUC1 O-glycosylation 910

MUC4 345

mucin 345

multicell layer 194

multicentric occurrence 1260

multidrug resistance 502, 934

multifocal low-grade urothelial cancer 260

mutation 778, 1416

Mycoplasma 949

$\mathrm{N}$-acetylglucosaminyltransferase V 1538

natural killer cells 539

neoadjuvant 1035

neoadjuvant chemoradiotherapy 1333

neoadjuvant chemotherapy 589

neoplastic syndromes 486

nested case-control study 426, 446

neuroblastoma 210, 238, 1416

neuron-glia-related cell-

adhesion molecule 531

neuropilins 1090

neutropenia 37

NF- $\kappa$ B 523, 659, 745, 1165

NHE 646

NK cytotoxicity 105

NK105 170

non-small-cell lung cancer 283, 741,1560

NSAIDs 557

nuclear localisation 1683

nucleoside analogue 628 nucleoside diphosphate kinase 1372

obesity 1005

occupational exposure 1300

octreotide 582

oesophageal cancer 123, 494, 868

oesophagus 1404

oestrogen receptor 58,659

offspring gender 688

oncogenes 1189

opioids 1523

OPN 1545

oral squamous cell carcinoma

$$
792
$$

osteonectin 1106

osteosarcoma 1552

outcome 1632

ovarian adenocarcinomas 1124

ovarian cancer $358,637,927$,

$1053,1291,1618$

ovarian low malignant potential

tumours 1124

overall response rate 1618

overrepresentation analysis 1432

oxaliplatin 593,862

oxaliplatin resistance 334

oxidative stress 1683

P-glycoprotein 322, 934

p16INK4A 1242

P2Y receptors 1372

p53 58, 1225, 1532, 1664

paclitaxel $43,170,888,1218$

palliative care 283,472

pancreatic cancer 464

pancreatic adenocarcinoma 598, 1106

pancreatic cancer $98,345,405$,

$$
\text { 523, 910, } 1432
$$

pancreatic neoplasms 1116

pangenomic analysis 238

panitumumab 1469

papillary thyroid carcinoma 531

papillomavirus 129

papillomavirus, human 989

parental occupation 1315

passive smoking 434

pathology 654

pathway analysis 792

patient recruitment 705

patient-centred assessment 1063

patient-reported outcomes 1469

PBD dimer 253

PDK-1 785

pegylated interferon 1532

pemetrexed 1071

peptides 1648,1655

performance status 458

pericellular proteolysis 201

peritoneal dissemination 550

pertuzumab 1338

PHA680632 1664

pharmacodynamic markers 1071 pharmacodynamics 290, 577

pharmacogenomics 145

pharmacokinetics 43, 290, 577

phase 0 trial 577

phase I 844, 1338

phase I study 170

phase I/II study 712

phase II study 862,1480

phosphorylated Akt 92

phosphorylated MAPK 92

photochemical internalisation 502

photodynamic therapy 502,

1381,1513

physical activity 995

PI3K/Akt 453

PIK3CD 1416

pineal 755

plasma 25-hydroxyvitamin D 446

plasma D-dimer 1053

plasma deoxynucleosides 1071

platelets 978

platinum chemotherapy 927

platinum-resistant 1618

platinum-sensitive 1618

PLD1 809

polymer micelles 170

polymorphisms $145,557,1175$, 1449

polyphenolic compounds 1372

polyunsaturated fat 1570

poor prognosis 792

poor-risk 162

population analysis 290

population dynamics 140

population study $29,112,730$

population-based cohort study 986

positive lymph nodes 605

post-cancer fatigue 612

postmenopausal 1486

postnatal vasculogenesis 513

poverty 140

PPAR 1388

predicting factor 405

predisposition 1305

preeclampsia 688

premenopausal 802

prenatal infection 992

preoperative 802

primary colon cancer cells 73

primary health care 486

primary therapy 802

probiotic 1028

profilin 1361

prognosis $1,37,238,247,523$,

$550,957,1211,1409,1690$

prognostic factors $302,391,605$, 952, 1538

prognostic marker 678, 730, 877

programmed cell death 619

progression-free survival (PFS)

1538,1618

proline-rich tyrosine kinase 2

(Pyk2) 50

promoter 277 promoter hypermethylation 1260

prostate cancer $378,557,730$, 826, 941, 952, 978, 1206, $1480,1613,1673,1683$

prostatic neoplasms 1690

proteasome inhibitor 1099

protein-truncating $B R C A 2$ mutations 826

proteomics 1116

PSA 678

psychosocial 1063

PTEN 1139

PTEN haploinsufficiency 678

PTHrP 183

pulmonary MALT lymphoma 949

pulmonary thromboembolism 1053

quality of life $283,1469,1625$

quantitative RT-PCR 531

\section{R306465 1344}

race 14,140

radiation 1058, 1505

radiation effects 115

radiation susceptibility 818

radiosensitivity 1696

radiotherapy (RT) 65, 115, 725

randomised trial 486, 1200

rapamycin 809

Ras signalling pathway 1425

RASSF2 1425

real-time methylation specific

PCR 1260

real-time PCR 1399

recurrence 50, 479

red meat 118

RelA 523

relative survival 999

renal cell cancer $112,429,1271$

resistance 919

rhabdomyosarcoma 785

risk 971, 1486

S-1 37, 458, 851

S100 267

S6 kinase 218

sarcoma 115, 1194

saturated fat 1570

SCC 1545

Scotland 999

screen-detected 725

screening 129, 971, 1493

second cancer 1058

second soft tissue sarcoma 695

SEER-Medicare 1606

self-report 14

senescence 941, 1225

sensitivity 1493

sequential chemotherapy 1613

seroepidemiologic studies 989

serum 778, 978

SFRPs 895

shared decision-making 6

signal transduction 368 
1728

single nucleotide polymorphism 832

siRNA 384

SJG-136 253

skin reactions 14

Smad-pathway 1388

small molecular inhibitor 785

small molecule 1344

small-cell lung cancer 162, 368, 1077

smoking 434, 1287

SNP 247

social contact 1315

social difficulties 1063

socioeconomic inequalities 999

socioeconomic status 140

SOCS proteins 231

solid tumours 539, 844

soluble interleukin-6 receptor 1513

somatostatin analogues 582

sorafenib 1480

spermatocytic seminoma 1707

squamous cell carcinoma 1404

St Gallen expert consensus meeting 654

stage II 1021

staging 868, 1393

STAT 378

STAT phosphorylation 231

stealth liposome 919

stellate cells 1106

stem cells 1189

stereology 1135

stromal 1194

subsite 700

superoxide dismutase 21116

surgery 1690 survival 283, 523, 670, 1124, 1266, 1567

symptom 1469

synergy 253, 628

synthetic vectors 210

T-cell signalling proteins 105

tailor-made therapy 145

tamoxifen 152, 327, 582

tea 1291

teenage and young adult cancer 1588

telomere 832, 1696

temozolomide 1225

TERF2 832

TERT 832

testicular germ cell tumour 1701, 1707

testicular microlithiasis 1701

TGF- $\alpha 1388$

TGF- $\beta$ signal 1388

the Sloane Project 725

three weekly schedule 1040

thymic epithelial tumour (TET) 22

thymidine phosphorylase 745

thymidylate synthase 334,1242 , 1449

thymidylate synthase inhibition 1071

thyroid cancers 818

time-dependent variable 37

TIMP 957

tissue microarray 785

titanocene 1234

TMX2-28 cells 809

TNM stage 1266

toll-like receptor 598

tonsil 670 topoisomerase II 58

total dietary fat 1570

toxicity 577,712

TP53 1157

trabectedin 1618

TRAIL 73

transcription 277

transformation 1457

transforming growth factor- $\beta$ 398, 1106

transforming growth factor- $\beta$ receptor 11175

trastuzumab 494

treatment 14

treatment decisions 6

treatment outcomes 717

treatment preference 717

treatment tradeoff method 717

trends 1009

treosulfan 1329

trichostatin A 562

trifunctional antibodies 315

tumour 277, 513

tumour angiogenesis 1372

tumour immunity 1655

tumour infiltrating lymphocytes

1135

tumour marker 412

tumour microenvironment 398 , 1106

tumour therapy 1271

tumoural invasion 957

twenty year follow-up 730

tyrosine kinase inhibitor 65, 453

tyrosine kinase receptor 857

UFT 297

undergraduate 472 urbanisation 140

uterine cancer 605

valproic acid 177

vascular disrupting 888

vascular endothelial growth

factor 391

VEGF 851

VEGF family 877

VEGF-C 1090

VEGF $_{165}$ b 223

$\mathrm{VEGF}_{x x x}$ b 223

vinorelbine 283

viral load 85

vitamin D 123

weekly docetaxel 1040

weekly epirubicin 1040

western blot 531

white cells 1266

WHO histologic classification 22

Wnt signalling 964

wound healing 1211

X-ray 1583

xanafide 58

XELOX 862

xenografts 73, 919

XR5944.14 844

young age 1046

ZD1839 183

ZD6126 888

ZD6474 934 